\title{
Etude de la production de vapeur alimentaire a la Société Béninoise des Brasseries (SOBEBRA)
}

\author{
Roger Gérard JOSSE ${ }^{1 *}$, Pierre DOSSOU YOVO ${ }^{2}$, Etienne SAGBO ${ }^{2}$, \\ Kouessi Joachim DALOHOUN ${ }^{3}$, Jacques FATOMBI ${ }^{2}$ et Nikita TOPANOU $^{2}$ \\ ${ }^{1}$ Laboratoire d'Analyses Physico-chimiques des Milieux Aquatiques APMIA/FAST/CHIMIE/UAC) 01 BP 526 \\ COTONOU (Bénin). \\ ${ }^{2}$ Laboratoire d'Expertise et de Recherche en Chimie de l'Environnement et de L'Eau \\ (LERCEE/FAST/CHIMIE/UAC) 01 BP 526 COTONOU (Bénin). \\ ${ }^{3}$ Laboratoire Central de la Société Béninoise des Brasseries (SOBEBRA) \\ *Auteur correspondant, E-mail : josserogergerard@yahoo.fr; 06 BP 2025 Cotonou, Bénin.
}

\section{RESUME}

L'objectif de cette étude est de mettre au point un système efficace de production de la vapeur alimentaire adapté aux conditions techniques et financières dans les entreprises des pays en voie de développement. A la SOBEBRA, le système de production est alimenté par l'eau de distribution et utilise un traitement externe (adoucissement par échange d'ions) et un traitement interne mixte qui se fait par la coagulation et par la dispersion. La vapeur est produite par une chaudière à tube alimentée par l'eau d'appoint et l'eau du retour condensât. Des échantillons sont prélevés aux différents points critiques de la chaîne de production afin de vérifier l'efficacité des traitements. Les résultats des analyses ont révélé que la dureté de l'eau d'appoint est stable et nulle. L'alcalinité est élevée dans la chaudière avec une valeur moyenne de pH 12. Le titre alcalimétrique (TA) et le titre alcalimétrique complet (TAC) sont aussi maintenus élevés dans la chaudière avec des valeurs moyennes respectives de $90^{\circ} \mathrm{F}$ et $120^{\circ} \mathrm{F}$. Dans la chaudière on note également l'augmentation des teneurs en phosphate et en silicate qui atteignent respectivement $400 \mathrm{mg} / \mathrm{L}$ et $59 \mathrm{mg} / \mathrm{L}$. Le dispositif de production satisfait aux normes internationales 3A. Le retour condensât est parfois contaminé par les opérations de lavage des bouteilles dans le terminal des opérations.

(c) 2011 International Formulae Group. All rights reserved.

Mots clés : vapeur alimentaire, adoucissement, traitement interne par dispersion, traitement interne par coagulation.

\section{INTRODUCTION}

La production de la vapeur alimentaire est très complexe et nécessite un équipement lourd et une technologie de pointe (Normes 3A; Afssan, 2005). Les industries alimentaires des pays en voie de développement ne disposant pas toujours de moyens techniques et financiers pour accéder à cette technologie, ont le plus souvent recours aux méthodes alternatives adoptées dans les pays du Nord. La composition physico-chimique d'une eau étant liée à la nature du terrain qu'elle traverse (Prasad et Narayana, 2004). Ces méthodes alternatives 
ne sont pas toujours adaptées aux types d'eau disponibles dans nos pays.

Ainsi se pose la problématique d'amélioration et d'adaptation des systèmes de production de la vapeur alimentaire aux conditions techniques, financières et géologiques dans les pays en voie de développement. Le faible niveau de développement et le nombre très limité de ces industries alimentaires font que le monde scientifique s'occupe très peu de leurs problèmes.

A l'échelle industrielle, les chauffages et les cuissons sont faits par la chaleur humide. L'industrie alimentaire doit prendre toutes les dispositions pour assurer une bonne qualité de la vapeur utilisée directement pour la production et la transformation de la nourriture (Miquel, 2003).

L'objectif général de cette étude est de combler ce vide scientifique qui s'observe dans le monde de l'industrie alimentaire dans nos pays, en recherchant les facteurs qui peuvent influencer la production de la vapeur alimentaire dans ces industries et en proposant des solutions pour la production de vapeur de qualité satisfaisante.

Nous avons étudié, dans ce cadre, le système de production de la vapeur alimentaire de la SOBEBRA, qui bénéficie de l'expertise d'un grand groupe international Baelen Gaillard Industrie (BGI).

Les objectifs spécifiques de cette recherche portent sur :

- La caractérisation physico-chimique des eaux aux différents points critiques de la chaîne de production de la vapeur ;

- La comparaison des résultats obtenus aux exigences normatives et règlementaires de la production de vapeur alimentaire (normes $3 \mathrm{~A}$ ) - Les propositions pour une amélioration du système.

\section{MATERIEL ET METHODES}

- La Société Béninoise des Brasseries (SOBEBRA) située à Cotonou, à $\mathrm{PK} 2,5$ route de Porto-Novo au Benin, est une usine de production de boissons (eaux minérales de Possotomé, boissons gazeuses et bières).
L'usine de Cotonou s'approvisionne en eau de distribution de la ville de Cotonou. Elle dispose d'une unité de production de la vapeur alimentaire (Figure 1).

- Ce système comporte un adoucisseur, un purgeur de vapeur associé à la chaudière, une bâche alimentaire et un retour condensât. La chaudière reçoit l'eau d'alimentation directement de la bâche alimentaire. La bâche alimentaire est à son tour chargée par l'eau condensée récupérée (eau du retour condensât) et par l'eau douce provenant de l'adoucisseur (eau d'appoint) ;

- L'adoucisseur est composé d'une résine échangeuse de cations $\mathrm{R}-\mathrm{SO}_{3} \mathrm{Na}$ et d'un réservoir de solution saturée de $\mathrm{NaCl}$ qui régénère la résine ;

- La vapeur produite sert au brassage de la bière, à la préparation de sirop des boissons gazeuses. Elle sert également au lavage des bouteilles, à la pasteurisation des boissons et au nettoyage des équipements (terminal des opérations).

- Le contrôle des paramètres se fait sur les échantillons prélevés au niveau des Points Critiques pour le Contrôle (CCP) de la chaîne de production de la vapeur de la SOBEBRA (Plusquellec, 2000) ;

- Les échantillons sont prélevés une fois par jour sur chacun des six CCP retenus ;

- Nous avons suivi la chaîne de production de la vapeur alimentaire de la SOBEBRA sur une période de 30 jours ;

- L'analyse statistique des données obtenues a été effectuée avec le logiciel Microsoft office Excel 2007. La variation des paramètres étant faible pendant la période d'étude, nous avons utilisé les moyennes obtenues pour la suite de l'étude.

- Les mesures sont réalisées à $20^{\circ} \mathrm{C}$;

- Le pH a été mesuré par un pH-mètre WTW LF 340 MERCK

- La température, la conductivité et le TDS ont été mesurés par un conductimètre WTW LF 320 MERCK ;

- Le Titre Alcalimétrique (TA) et le Titre Alcalimétrique Complet (TAC) ont été déterminés par titrimétrie (Matini et al., 2009) ; 
- Le Titre Hydrotimétrique (TH) a été déterminé par titrimétrie (méthode complexométrique) (Matini et al., 2009);

- Les phosphates et les silicates ont été dosés par colorimétrie à l'aide du Kit aquamerk tests phosphates et silicates de référence : 1.08021.0001 Merck (Raposo et al., 2008)

- Les sulfites sont dosés par colorimétrie avec le Kit aquamerk sulfite test de référence 1.11148.0001.

\section{RESULTATS}

L'eau d'appoint du système de production de la vapeur alimentaire doit être exempte de tout produit coloré, toxique ou odorant pouvant être libéré en amont de la production (Klemes et al., 2008). L'eau utilisée doit permettre l'échange continu de chaleur, la protection à la corrosion et à l'entartrage et la production de haute qualité de vapeur (Symposium International QUALIMA 01).

Le Tableau 1 présente les différentes valeurs des paramètres physico-chimiques de l'eau d'alimentation. Nous notons que l'eau d'alimentation est faiblement acide et son $\mathrm{pH}$ tend vers la neutralité $(\mathrm{pH}=6,4)$. Le TA est nul ; le TAC est faible avec une moyenne de $21{ }^{\circ} \mathrm{F}$ tandis que le $\mathrm{TH}$ est relativement élevé $\left(27{ }^{\circ} \mathrm{F}\right)$ ce qui justifie sa faible minéralisation avec une conductivité moyenne de $136 \mu \mathrm{S} / \mathrm{cm}$ à $20{ }^{\circ}$ C. Selon les recommandations des Normes 3A (principales normes américaines en matière d'équipement), l'eau d'appoint doit être douce. Le traitement externe ici a consisté à adoucir l'eau d'alimentation. Les analyses ont révélé que la dureté de l'eau de l'adoucisseur (eau après le traitement externe) est constante et nulle sur toute la période d'étude

Le Tableau 2 présente les paramètres physico-chimiques de l'eau le long de la chaîne de production de la vapeur. On note une concentration en phosphate nulle dans les eaux de ville, de l'adoucisseur, du retour condensât et du collecteur vapeur. Ce taux est uniquement élevé dans la chaudière. Ce taux élevé des phosphates dans la chaudière maintient les particules en suspension dans la chaudière. Les phosphates forment des particules dispersées de boue (energyconcepts.tripod.com).

Les valeurs moyennes du $\mathrm{pH}$ dans l'eau d'alimentation et dans l'eau de l'adoucisseur sont inférieures à 7. Par contre dans l'eau de retour condensât, dans la bâche alimentaire, la chaudière et le collecteur vapeur, le $\mathrm{pH}$ est basique et admet un maximum ( $\mathrm{pH} 12)$ dans l'eau de la chaudière. Le $\mathrm{pH}$ du collecteur vapeur est en moyenne 8 tandis que celui du retour condensât est en moyenne 9,5. On note un écart d'environ deux unités de $\mathrm{pH}$ entre celui du retour condensât et du collecteur vapeur. Cette différence des valeurs du $\mathrm{pH}$ pourrait s'expliquer par une contamination de la vapeur dans le terminal des opérations (Figure1).

Les valeurs de TA de l'eau de ville et de l'adoucisseur sont nulles. Dans les eaux du retour condensât, de la bâche alimentaire, de la chaudière et du collecteur vapeur, nous notons une variation en dents de scie des valeurs de TA avec un pic $\left(\mathrm{TA}=90^{\circ} \mathrm{F}\right)$ dans l'eau de la chaudière dû à un apport d'alcalinité sodique. Ce traitement maintient les valeurs du TA, du TAC et du pH élevées dans la chaudière.

Les valeurs du TAC (Figure 5) présentent des fluctuations sur toute la chaîne de production de la vapeur et admettent un maximum au niveau de la chaudière. Ce qui confirme les résultats des Figures 3 et 4 . Ces valeurs restent relativement élevées dans l'eau du retour condensât et dans la chaudière. Les courbes du TA et du TAC ont sensiblement la même allure. Les valeurs du TA et du TAC du collecteur vapeur sont plus faibles que celles du retour condensât, ce qui justifie l'écart du pH observé entre le collecteur vapeur et le retour condensât au niveau de la Figure 3.

La teneur en sulfites est faible sur toute la chaîne de production (inférieur à $1 \mathrm{mg} / \mathrm{L}$ ). On remarque par contre une augmentation remarquable de cette teneur dans l'eau de la chaudière où elle est relativement élevée et atteint $4 \mathrm{mg} / \mathrm{L}$ (Figure 6). Cette élévation serait due à l'utilisation des réducteurs 
(sulfites) dans la chaudière pour l'élimination de l'oxygène dissout.

La Figure 8 fait apparaitre des taux de TDS dans la chaîne de production de la vapeur. On observe une valeur maximale dans les eaux de retour condensât et de la chaudière. $\mathrm{Au}$ cours $\mathrm{du}$ traitement par coagulation, le silicate de sodium est utilisé pour réagir sélectivement avec la dureté en magnésium. Le bicarbonate de calcium existant dans l'eau d'appoint est traité par les cendres caustiques pour former le carbonate de calcium et le carbonate de sodium. Le carbonate de calcium est relativement insoluble et tend à précipiter. Les températures élevées de la chaudière empêchent la précipitation du carbonate sur le métal de la chaudière sous forme de tartre. Le traitement par coagulation maintient les valeurs du $\mathrm{pH}$, du TA et du TAC élevées dans la chaudière de même que la concentration en silicates de sodium. Ce qui confirme les résultats des Figures 2, 3, 4 et 5.

La conductivité est l'aptitude d'une eau à permettre le passage du courant électrique. Elle exprime le degré d'ionisation de l'échantillon selon Mor et al. (2006). La conductivité de l'eau d'alimentation, de l'adoucisseur et celle du collecteur vapeur est faible (moins de $170 \mu \mathrm{S} / \mathrm{cm}$ ). Celle du retour condensât atteint les $800 \mu \mathrm{S} / \mathrm{cm}$ et celle de la bâche alimentaire avoisine les $600 \mu \mathrm{S} / \mathrm{cm}$. Elle est plus élevée au niveau de la chaudière avec une moyenne de $1300 \mu \mathrm{S} / \mathrm{cm}$ (Figure 8).

Tableau 1 : paramètres physico-chimiques de l'eau d'alimentation.

\begin{tabular}{lccc}
\hline Paramètres & Moyenne & Ecart-type & Nombre d'échantillons \\
\cline { 2 - 4 } & 6,4 & 0,6 & 30 \\
$\mathrm{pH}$ & 0 & 0 & 30 \\
$\mathrm{TA}\left({ }^{\circ} \mathrm{F}\right)$ & 21 & 1,6 & 30 \\
$\mathrm{TAC}\left({ }^{\circ} \mathrm{F}\right)$ & 27 & 2,05 & 30 \\
$\mathrm{TH}\left({ }^{\circ} \mathrm{F}\right)$ & 105 & 12 & 30 \\
$\mathrm{TDS}(\mathrm{mg} / \mathrm{L})$ & & & 30 \\
Conductivité & 136 & 27 & \\
$\left(\mu \mathrm{S} / \mathrm{cm}\right.$ à $\left.20{ }^{\circ} \mathrm{C}\right)$ & & & \\
\hline
\end{tabular}

Tableau 2: Paramètres physico-chimiques de l'eau le long de la chaîne de production de la vapeur.

\begin{tabular}{|c|c|c|c|c|c|c|}
\hline Paramètres & Ville & Adoucisseur & $\begin{array}{l}\text { Retour } \\
\text { condensât }\end{array}$ & $\begin{array}{c}\text { Bâche } \\
\text { alimentaire }\end{array}$ & Chaudière & $\begin{array}{c}\text { Collecteur } \\
\text { vapeur }\end{array}$ \\
\hline $\mathrm{pH}$ & $6,4 \pm 0,6$ & $6,53 \pm 0.6$ & $9,5 \pm 2$ & $8,5 \pm 2$ & $12 \pm 1$ & $8 \pm 2$ \\
\hline TA $\left({ }^{\circ} \mathrm{F}\right)$ & 0 & 0 & $30 \pm 15$ & $20 \pm 10$ & $90 \pm 30$ & $10 \pm 5$ \\
\hline TAC $\left({ }^{\circ} \mathrm{F}\right)$ & $21 \pm 1,6$ & $25 \pm 2$ & $43 \pm 20$ & $40 \pm$ & $120 \pm 12$ & $30 \pm 9$ \\
\hline $\mathrm{TH}\left({ }^{\circ} \mathrm{F}\right)$ & $27 \pm 2$ & 0 & 0 & 0 & 0 & 0 \\
\hline TDS (mg/L) & $105 \pm 12$ & $100 \pm 25$ & $152 \pm 33$ & $100 \pm 38$ & $240 \pm 50$ & $30 \pm 12$ \\
\hline Conductivité & $136 \pm 27$ & $180 \pm 20$ & $800 \pm 100$ & $600 \pm 80$ & $1300 \pm 190$ & $170 \pm 60$ \\
\hline Sulfites $(\mathrm{mg} / \mathrm{L})$ & $0,2 \pm 0$ & $0,2 \pm 0$ & $0,3 \pm 0.1$ & $0,2 \pm 0.1$ & $4 \pm 0.5$ & $0,5 \pm 0.1$ \\
\hline Silicates $(\mathrm{mg} / \mathrm{L})$ & $8 \pm 0.1$ & $8 \pm 0.1$ & $37 \pm 0.9$ & $28 \pm 0.5$ & $59 \pm 0.9$ & 0 \\
\hline $\begin{array}{l}\text { Phosphates } \\
\text { (mg/L) }\end{array}$ & 0 & 0 & 0 & 0 & $400 \pm 50$ & 0 \\
\hline
\end{tabular}




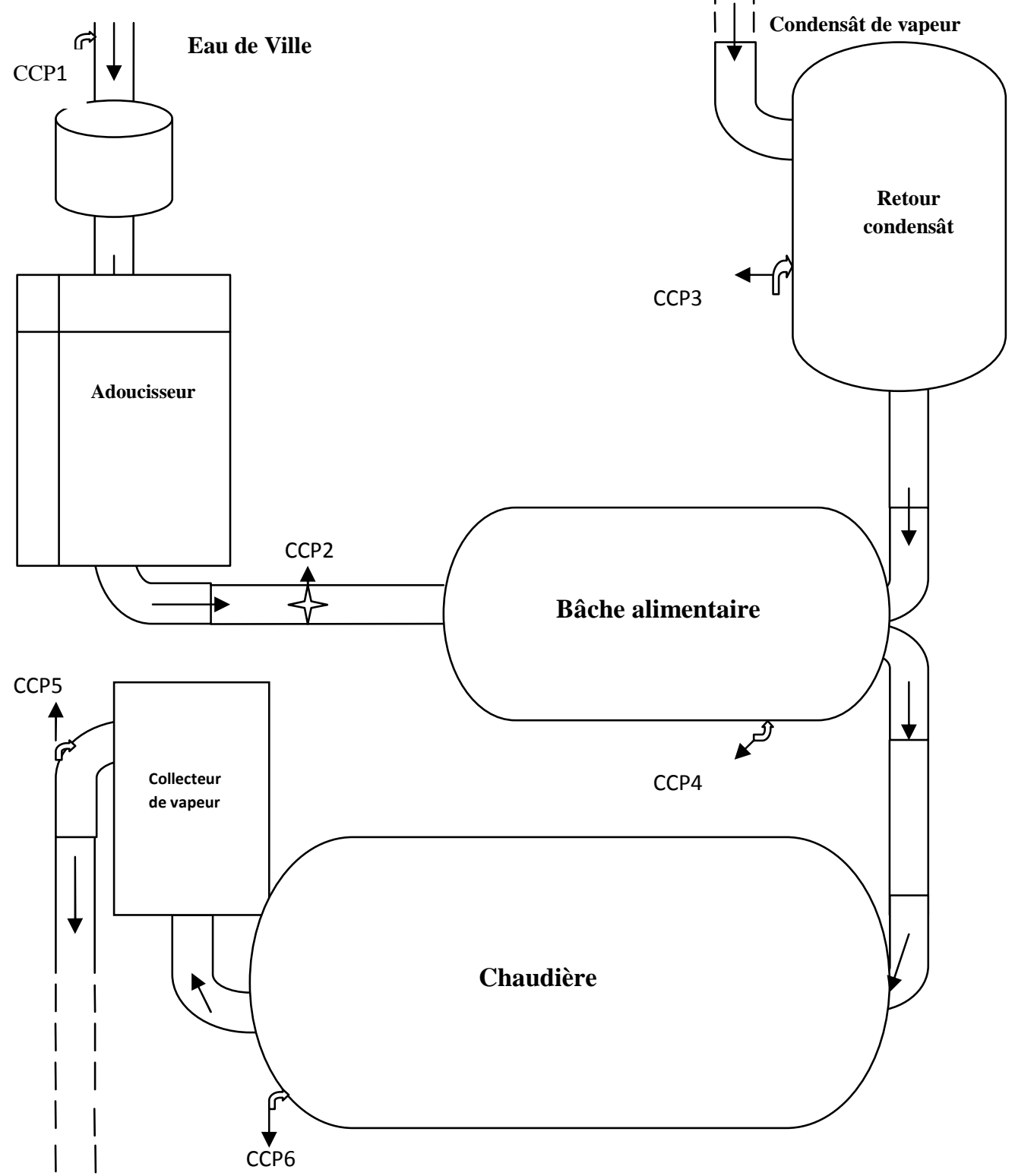

Terminal des opérations.

Figure 1 : Dispositif de production de la vapeur alimentaire.

$\mathrm{CCP} 1$ : point de prélèvement eau de ville $; \mathrm{CCP} 2$ : point de prélèvement adoucisseur ; $\mathrm{CCP} 3$ : point de prélèvement retour condensât ; CCP4 : point de prélèvement bâche alimentaire ; CCP5 : point de prélèvement collecteur de vapeur ; CCP6 : point de prélèvement chaudière. 


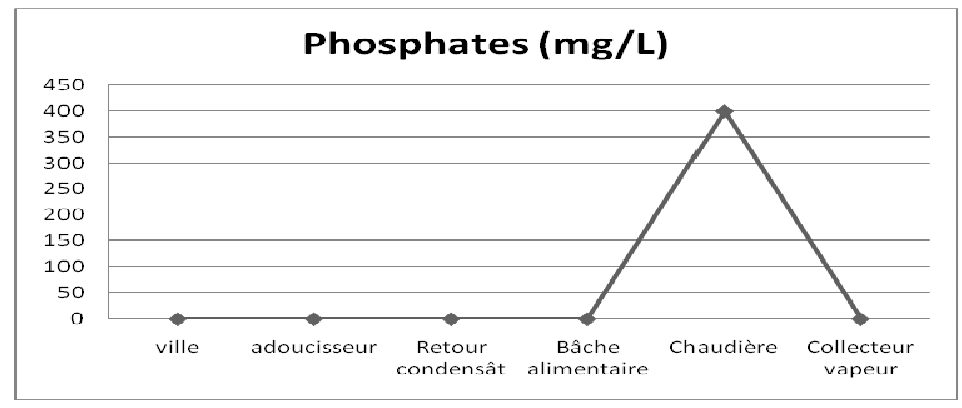

Figure 2 : Variation de la teneur en phosphates dans la chaîne de production de la vapeur alimentaire (en $\mathrm{mg} / \mathrm{L})$.

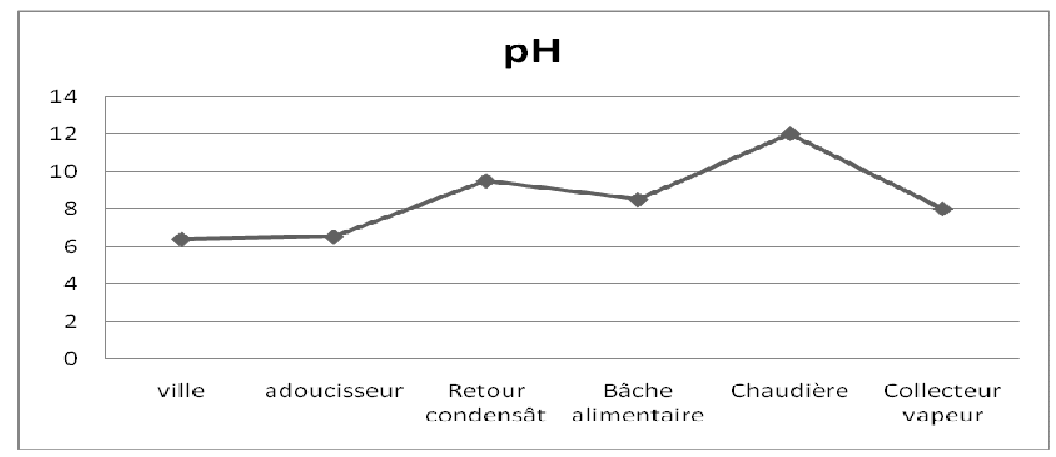

Figure 3 : Variation du pH dans la chaîne de production de la vapeur alimentaire.

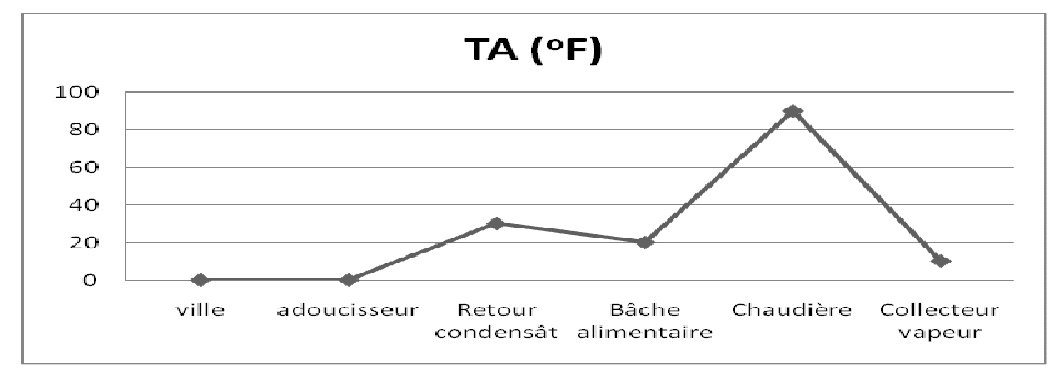

Figure 4 : Variation du TA dans la chaîne de production de la vapeur alimentaire $\left(\mathrm{en}{ }^{\circ} \mathrm{F}\right)$.

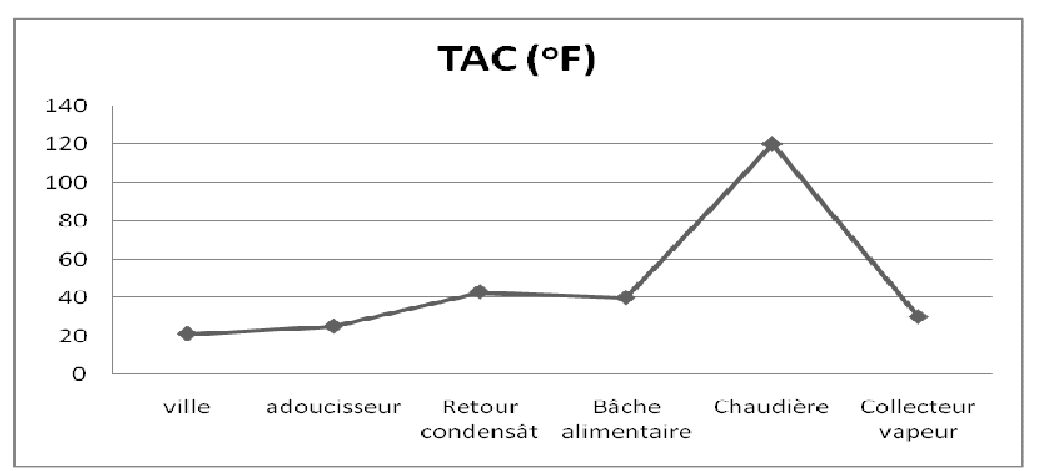

Figure 5 : Variation du TAC dans la chaîne de production de la vapeur alimentaire $\left(\mathrm{en}{ }^{\circ} \mathrm{F}\right)$. 


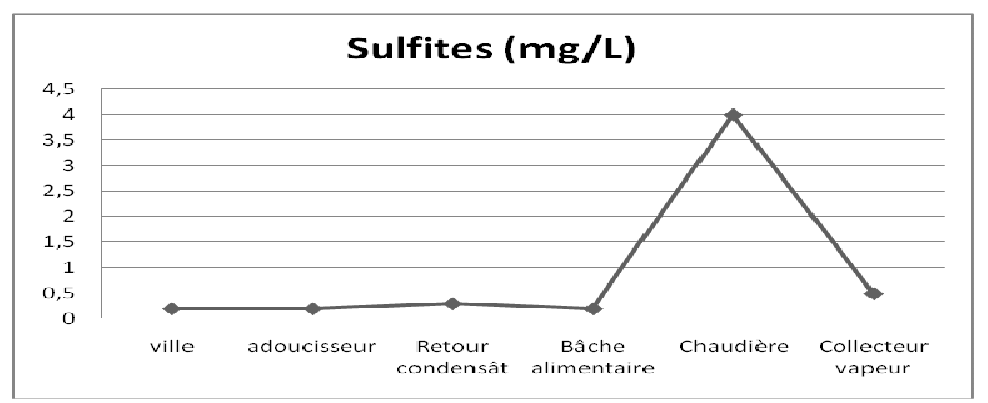

Figure 6 : Variation de la teneur en sulfites dans la chaîne de production de la vapeur alimentaire (en $\mathrm{mg} / \mathrm{L})$.

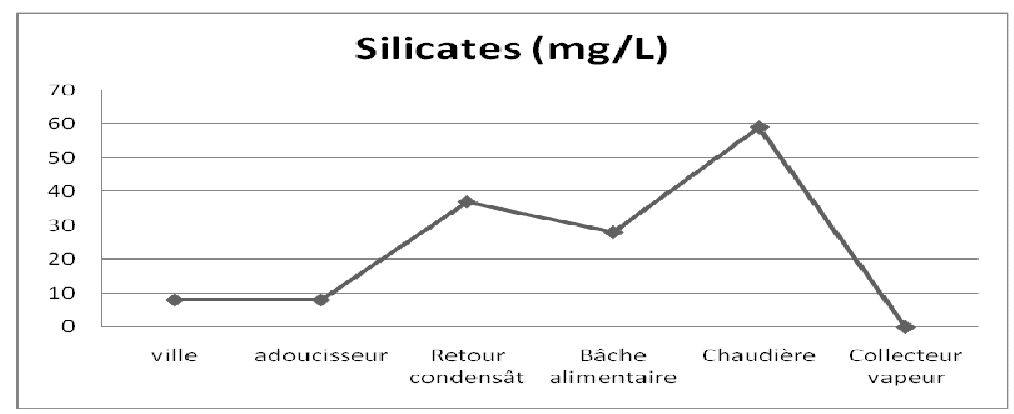

Figure 7 : Variation de la teneur en silicates dans la chaîne de production de la vapeur alimentaire (en $\mathrm{mg} / \mathrm{L})$.

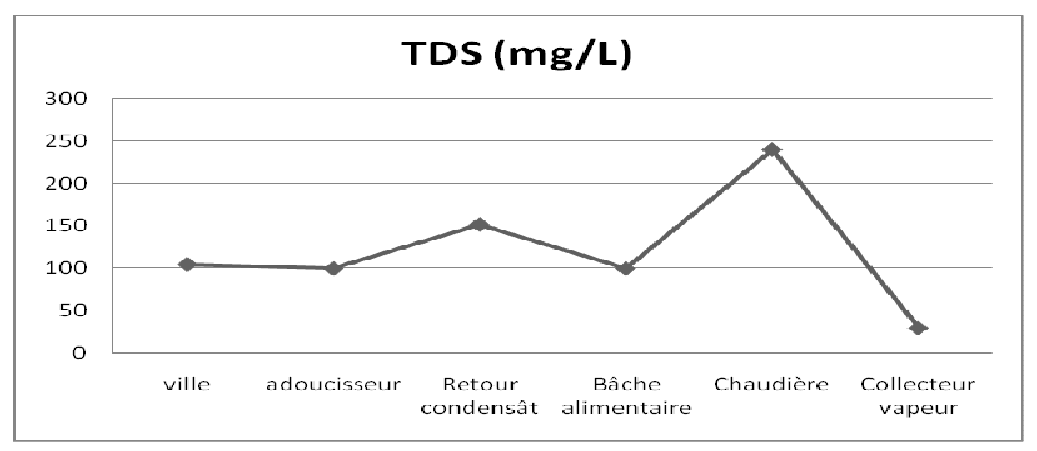

Figure 8 : Variation de TDS dans la chaîne de production de la vapeur alimentaire (en mg/L).

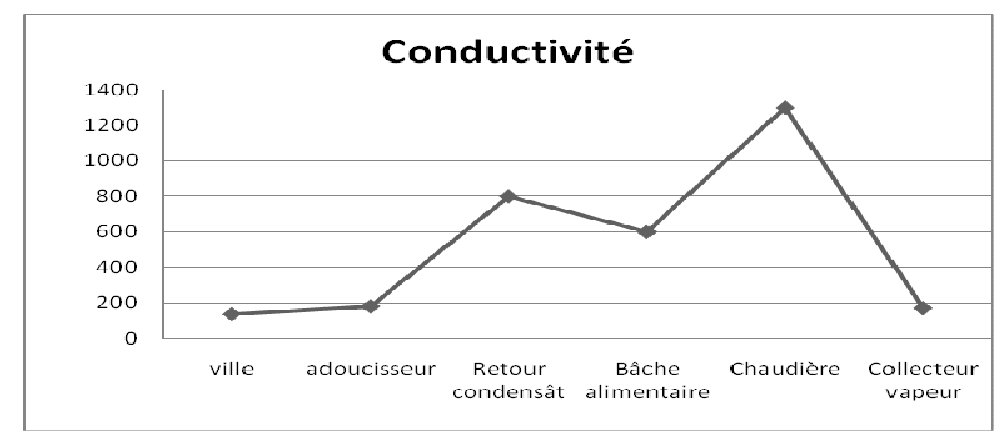

Figure 9 : Variation de la conductivité dans la chaîne de production de la vapeur alimentaire (en $\mu \mathrm{S} / \mathrm{cm}$ à $20^{\circ} \mathrm{C}$ ). 


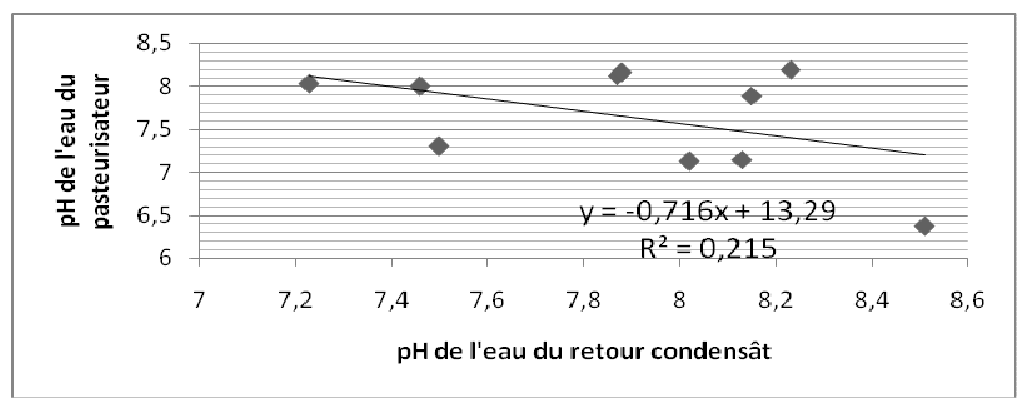

Figure 10 : Corrélation entre le pH de l'eau du pasteurisateur et celui de l'eau du retour condensât.

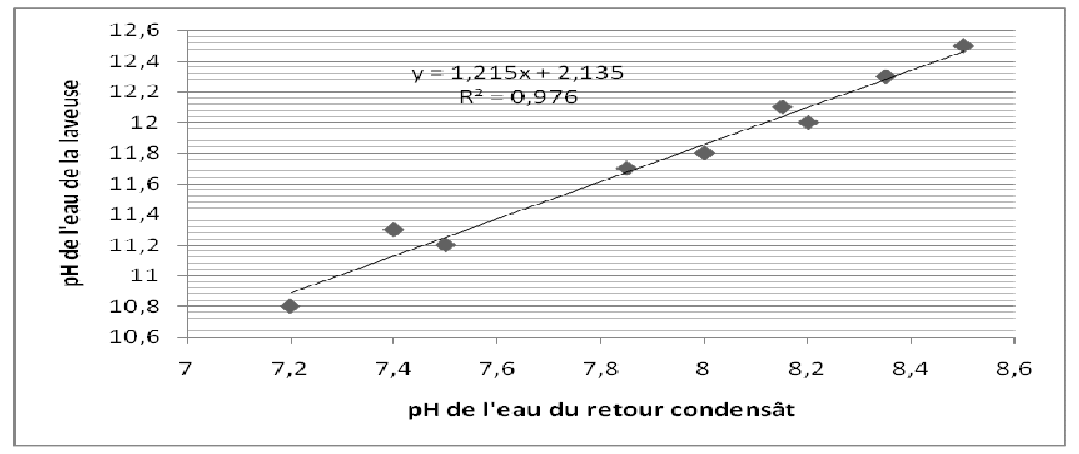

Figure 11 : Courbe de la corrélation entre le pH de l'eau de la laveuse et celui de l'eau du retour condensât.

\section{DISCUSSION}

Le traitement externe qui consiste à éliminer la dureté totale élevée dans l'eau de l'alimentation (Tableau1) utilise un adoucisseur composé de résines $\mathrm{R}-\mathrm{SO}_{3} \mathrm{Na}$ et d'un dispositif contenant une solution saturée de chlorure de sodium $(\mathrm{NaCl})$ pour régénérer les résines lorsque celles-ci sont saturées. Les ions calcium $\left(\mathrm{Ca}^{2+}\right)$ et magnésium $\left(\mathrm{Mg}^{2+}\right)$ responsables de la dureté totale de l'eau sont échangés lors de leur passage sur la résine par les ions $\mathrm{Na}^{+}$. L'eau d'appoint est donc constamment douce ; ce qui est conforme aux recommandations des Normes $3 \mathrm{~A}$.

La concentration en solides dissous augmente dans la chaudière avec la production de la vapeur. Il en résulte un faible transfert de chaleur ce qui réduit l'efficacité de la chaudière. Les gaz dissous tels que l'oxygène et le dioxyde de carbone entraînent la corrosion des métaux de la chaudière. L'oxygène se combine avec le fer pour former le dihydroxyde de fer (II), le dioxyde de carbone et l'oxygène attaquent le fer pour donner le carbonate de fer $\left(\mathrm{FeCO}_{3}\right)$. Afin de protéger la chaudière de ces contaminants, ils sont contrôlés ou éliminés, grâce à un traitement interne. Le traitement interne (traitement chimique à l'intérieur de la chaudière) est donc réalisé pour :

- Réagir avec une éventuelle dureté de l'eau d'appoint afin de l'empêcher de précipiter avec le métal de la chaudière (tartre) ;

- Conditionner des matières en suspension telles les boues de dureté ou l'oxyde de fer afin de les rendre non-adhérentes au métal de la chaudière ;

- Assurer une protection anti-moussage pour permettre une concentration raisonnable en solides dissous ou en suspension dans la chaudière sans risque de primage;

- Eliminer l'oxygène de l'eau et assurer une alcalinité suffisante pour empêcher la corrosion dans la chaudière (www.thermidaire.con). Ce traitement est fait par adjonction de produits chimiques. 
Il existe deux approches générales pour le traitement interne: le traitement par coagulation et le traitement par dispersion. Lorsque la quantité totale de boue est élevée (dureté élevée de l'eau d'appoint), il est préférable de coaguler les boues pour former des particules floculeuses qui seront éliminées par vidange. Quand la quantité de boue n'est pas trop élevée (faible dureté de l'eau d'appoint) on utilise généralement le traitement par dispersion. Ce traitement consiste à utiliser une quantité importante de phosphates dans le traitement, ce qui maintient la concentration en phosphate élevée dans l'eau de la chaudière. Le système étudié utilise un système de traitement interne mixte.

L'analyse des Figures 3, 4 et 5 révèle que les valeurs du $\mathrm{pH}$, du TA et du TAC sont particulièrement élevées dans la chaudière. Ces valeurs résultent du traitement interne par conditionnement en utilisant un complexe chimique composé de phosphate, de soude et de sulfites (Water Environment Federation 2005).

La Figure 8 fait apparaître des taux de TDS dans la chaîne de production de la vapeur. On observe une valeur maximale dans les eaux de retour condensât et de la chaudière.

Les résultats obtenus montrent que le traitement interne utilisé à la SOBEBRA, (traitement interne mixte utilisant la coagulation et la dispersion) est une méthode adaptée et très efficace.

Le retour condensât est un condensât de vapeur (Règlement $\mathrm{CE} \mathrm{N}^{\circ}$ 852). Les valeurs de la conductivité, du TA, du TAC et $\mathrm{du}$ TDS obtenues révèlent que l'eau du retour condensât est chargée en ions. Ces valeurs pour l'eau de l'adoucisseur sont faibles de même que celles du collecteur vapeur. L'hypothèse selon laquelle l'eau du retour condensât serait contaminée par le terminal des opérations est alors évidente. Pour déterminer l'origine de cette contamination, nous avons étudié la corrélation entre les différents CCP susceptibles de contaminer l'eau de retour condensât.
Le terminal des opérations étant l'ensemble des opérations de préparation des boissons (bières et boissons gazeuses), de la pasteurisation et du lavage, nous chercherons celles qui seraient à l'origine de la contamination. Les boissons étant acides, la contamination par les opérations de préparation n'est pas envisageable car on observe une augmentation du $\mathrm{pH}$.

L'étude de la corrélation entre deux séries permet d'identifier la dépendance ou la non dépendance qui existe entre ces deux séries. Le degré de dépendance est exprimé en calculant le coefficient de corrélation et en traçant la droite d'ajustement. Plus le coefficient de corrélation se rapproche de 1 plus la corrélation est forte.

La Figure 10 exprime la variation du $\mathrm{pH}$ de l'eau du retour condensât en fonction $\mathrm{du} \mathrm{pH}$ de l'eau du pasteurisateur. La droite obtenue correspond à une faible corrélation avec des points dispersés autour de la droite et un coefficient faible $\left(\mathrm{R}^{2}=0,215\right)$. Ces résultats montrent que la contamination de l'eau du retour condensât ne provient pas du pasteurisateur. Nous avons également recherché la probable contamination du retour condensât par le lavage des bouteilles. La Figure 11 présente la courbe de la corrélation entre le $\mathrm{pH}$ de l'eau du collecteur vapeur et celui de l'eau du premier bac de lavage (le bac le plus concentré en soude de la laveuse). La droite obtenue correspond à une forte corrélation avec des points condensés autour de la droite et un coefficient fort $\left(\mathrm{R}^{2}=0,976\right)$. Le retour condensât est donc alors contaminé par la laveuse.

Lors de l'utilisation de la vapeur, une partie de celle-ci se condense et est recueillie par le retour condensât. Au cours des opérations de lavage, la vapeur entre en contact avec les eaux de la laveuse qui la contaminent. Le lavage des bouteilles se faisant en milieu basique (solution de soude caustique de 2 à $4 \mathrm{mg} / \mathrm{L}$ ) expliquerait la valeur élevée du $\mathrm{pH}$ de l'eau du retour condensât qui est contaminée au cours de cette opération de lavage. 


\section{Conclusion}

Les résultats obtenus au cours de ces travaux montrent que le système de production de la vapeur alimentaire étudié utilise un traitement externe et un traitement interne. Le traitement externe (adoucissement) élimine complètement la dureté de l'eau d'appoint. Le traitement interne combine la coagulation et la dispersion. Le dispositif de production satisfait aux normes internationales $3 \mathrm{~A}$, mais ne dispose pas de moyens pour contrôler la qualité finale de la vapeur produite. L'efficacité des traitements externe et interne garantit une bonne qualité de la vapeur produite. Afin de s'assurer en permanence d'une meilleure qualité de vapeur produite, il serait préférable de ne plus recueillir le condensât de l'opération de lavage des bouteilles.

\section{REFERENCES}

Afssan. 2005. Saisine n ${ }^{\circ}$ 2002-SA-0317 relatif à l'emploi de diverses substances dans l'eau des chaudières fournissant de la vapeur d'eau destinée à entrer au contact direct avec les denrées alimentaires. Maisons-Alfort.

http://www.energyconcepts.tripod.com/energy concepts/water_treatment .htm.

http://www.thermidaire.on.ca/boilerfeed.html.

Klemes J, Smith R, Kim JK. 2008. Handbook of Water and Energy Management in Food Processing ( $1^{\mathrm{e}}$ édn). Woodhead Publishing Limited: England.

Les Normes 3A: les principales normes américaines en matière d'équipements, établies par les comités spécialisés de l'International Association of Food Industry Suppliers; la norme $3 \mathrm{~A} \quad \mathrm{~N}^{\circ}$ 609.01 .

Matini L, Moutou JM, Kongo-Mantono MS. 2009. Evaluation hydro-chimique des eaux souterraines en milieu urbain au
Sud-Ouest de Brazzaville. Afrique Science, 05(1): 82 - 98.

Miquel G. 2003. La qualité de l'eau et de l'assainissement en France. Rapport du Sénat $\mathrm{N}^{\mathrm{o}} .215$ de l'Office parlementaire d'évaluation des choix scientifiques et technologiques.

Mor S, Ravindra K, Dahiya RP, Chandra A. 2006. Leachate characterization and assessment of groundwater pollution near municipal solid waste landfill site. Environ. Monit. Assess., 118(2): 435-456.

Plusquellec. 2000. Les eaux. In Techniques d'Analyse et de Contrôle dans les Industries Agroalimentaires ( $3^{\mathrm{e}}$ édn). APRIA: France; 327-334.

Prasad BG, Narayana TS. 2004. Subsurface water quality of different sampling stations with some selected parameters at Machilipatnam Town. Nat. Env. Poll. Tech., 3(1): 47-50.

Qualima01 - Tlemcen. 2004. Symposium International : Qualité et Maintenance au Service de l'Entreprise.

Raposo F, de la Rubia MA, Borja R, Alaiz M. 2008. Assessment of a modified and optimised method for determining chemical oxygen demand of solid substrates and solutions with high suspended solid content. Talanta, 76(2): 448-453.

Règlement (CE) $\mathrm{N}^{\circ}$ 852/2004 du parlement européen et du conseil du 29 avril 2004 relatif à l'hygiène des denrées alimentaires.

Water Environment Federation. 2005. Standard Methods for the Examination of Water and Wastewater ( $21^{\text {st }}$ edn). APHA: Washington. 